\title{
Genomic characterization of five commonly used endometrial cancer cell lines
}

\author{
ERIC J. DEVOR ${ }^{1,2}$, JESUS GONZALEZ-BOSQUET ${ }^{1,2}$, KRISTINA W. THIEL ${ }^{1}$ and KIMBERLY K. LESLIE ${ }^{1,2}$ \\ ${ }^{1}$ Department of Obstetrics and Gynecology, University of Iowa Carver College of Medicine; \\ ${ }^{2}$ Holden Comprehensive Cancer Center, University of Iowa Hospitals and Clinics, Iowa City, IA 52242, USA
}

Received June 26, 2020; Accepted September 22, 2020

DOI: 10.3892/ijo.2020.5139

\begin{abstract}
Recently, the compilation of massive amounts of genetic and genomic information on a wide variety of human cancer types, collectively known as The Cancer Genome Atlas (TCGA), has revealed a wealth of descriptive classification schemes both within and between different types and sources of cancer. In endometrial cancer, TCGA analyses have produced a post hoc scheme composed of four clusters: DNA polymerase $\varepsilon$ catalytic subunit A (POLE) ultra-mutated (cluster 1), microsatellite instability (MSI) hypermutated (cluster 2), copy-number low (endometrioid, cluster 3 ) and copy-number high (serous-like, cluster 4). Given that cultured cells are the pre-clinical platform of cancer research, it was questioned how representative endometrial cancer cultured cell lines are in the context of TCGA-driven classification scheme. To address this issue in endometrial cancer cell lines, the present study investigated five commonly used cell lines: Ishikawa, ECC-1, Hec50co, KLE And RL95-2. The histology, mutation profile, MutL homolog 1 promoter methylation, copy-number variation, homologous recombination repair and microsatellite instability in each of these cell lines was assessed. The result of this characterization was that none of the cell lines fits neatly into any one of TCGA classes but are still useful models for groups of endometrial tumors. Furthermore, the contention that the ECC-1 cell line is actually Ishikawa was addressed using additional data. It was confirmed that ECC-1 cells likely no longer exist as ECC-1 but that they are not exactly Ishikawa either. For this reason, ECC-1 cells are suggested to be used in vitro but with this caveat in mind. Finally, we compiled a database of 127 endometrial cancer cell lines, including the five reported on here. The wide range of variation found in these cell lines highlights the need to further characterize these cells to select models that are more representative of
\end{abstract}

Correspondence to: Dr Kimberly K. Leslie, Department of Obstetrics and Gynecology, University of Iowa Carver College of Medicine, 200 Hawkins Drive, Iowa City, IA 52242, USA

E-mail: kimberly-leslie@uiowa.edu

Key words: endometrial cancer, cell lines, genomics, The Cancer Genome Atlas the various histological and genomic aspects of endometrial cancer.

\section{Introduction}

Endometrial cancer ranks as the most common gynecological cancer and the fourth most common cancer among women worldwide (1). Estimates for the United States predicted that there would be $\sim 62,000$ new cases of endometrial cancer with $>12,000$ deaths in 2019 (2). Mortality from endometrial cancer in the United States will be almost equal to that from ovarian cancer at $\sim 14,000$ deaths per year and, at any one time, an excess of 700,000 women are living with endometrial cancer in the United States alone (2). Endometrial cancer incidence as well as recurrence risk is on the rise, with a growing disparity in outcomes among ethnic groups $(3,4)$. For example, the 5-year survival rate is only $64 \%$ for non-Hispanic Black women in the United States compared with $86 \%$ for non-Hispanic white women, a $22 \%$ difference (3). Thus, it is important to improve our understanding of these trends and seek ways to address them clinically. One avenue that should be explored is new therapeutic strategies involving combinations of agents, which must begin with in vitro studies using endometrial cancer cell lines.

Beginning with the HeLa cell line in the 1950s (5), cultured cancer cells have been the workhorse of cancer research. While these cells have been the source of numerous breakthroughs in our understanding of cancer biology and have served as the front lines of cancer treatment discovery, there have been contamination with other cell lines, misidentification of cultured cells and reversals in reports of contamination with other cell lines that have caused some to question their utility as cancer models, particularly their clinical relevance $(6,7)$. However, with the development of next generation genetic and genomic technologies, it has become possible to fully characterize cancer cell lines in order to objectively evaluate how representative they are. For example, four studies of ovarian cancer cell lines have provided a wealth of information for a total of 108 different cell lines (8-11). There are numerous ovarian cancer cell lines and only six of them were represented in all four studies and only eight more were found in three of the four. Similarly, while there are $>100$ uterine cancer cell lines, only a few are routinely used and none have been sufficiently characterized 
to assess their status as representatives of patients diagnosed with endometrial cancer.

To assess how well individual endometrial cancer cell lines represent larger groups of patient tumors, the present study selected the five of the most commonly used endometrial cancer cell lines: Ishikawa, ECC-1, KLE, RL95-2 And Hec50co. Specific analyses included histology, mutation screening, MutL homolog (MLH)1 promoter methylation, homologous recombination repair (HRR), copy number variation (CNV) and microsatellite instability (MSI) (12-16). The issue of what ECC-1 cells really are, as several studies have suggested that they are actually Ishikawa cells and that ECC-1 cells no longer exist $(8,17,18)$, was also investigated. The current data support this view but also suggested that there are enough differences between Ishikawa and ECC-1 cells to regard ECC-1 as a useful cell line. Finally, the present study assembled a database listing a total of 127 endometrial cancer cell lines, some of which are well known but several are not. If novel and more representative pre-clinical endometrial cancer models are identified, then these and other lines should be examined to the extent that Ishikawa, ECC-1, Hec50co, KLE and RL95-2 cells are in the current study.

\section{A brief history of the five cell lines}

Ishikawa. Dr Masato Nishida first established the classic Ishikawa cell line in 1980 (16). Dr Nishida recognized that no one cell line could possess all of the relevant characteristics of a cancer and that there were few endometrial cancer cell lines available. Ishikawa cells were derived from a 39-year-old Japanese patient who presented with a well differentiated stage 2 endometrial adenocarcinoma. The cell line was readily established and propagated and maintained both estrogen and progesterone receptors in culture. Since their original establishment, Ishikawa cells have been used as a model for type I endometrial cancer (11) in labs around the world and have appeared in $>500$ publications based on a PubMed (pubmed. ncbi.nlm.nih.gov/) search using the search term 'Ishikawa.' In 2002, Dr Nishida reviewed the history of the cell line and noted that the original parent cells were distributed to other labs but that sub-clones were developed in 1993 (19-21). These were distributed after 1993, with sub-clone 3-H-4 being the most commonly used line between 1993 and 1996; sub-clone 3-H-12 was the most used line after 1996 (19-21).

ECC - 1. This cell line was developed by the late P.G. Satyaswaroop by passaging cells from a well differentiated endometrial adenocarcinoma from a 68 -year-old patient into the infrascapular region of ovarectomized Balb/c (nu/nu) mice (22). The cells were subsequently cultured in vitro and maintained using both estrogen and progesterone receptors in culture. Moreover, ECC-1 cells express proteins characteristic of luminal epithelium upon stimulation with EGF (23). In general, ECC-1 cells were regarded as most similar to Ishikawa cells, though there were useful unique features, including the luminal epithelium characteristics, that differentiate ECC-1 cells from the more glandular epithelial characteristics of other endometrial cancer cell lines, such as Ishikawa (23). This cell line was deposited in the American Type Culture Collection (ATCC) but is no longer available as the ATCC no longer recognizes it as a unique cell line based upon work by
Korch (8). ATCC (www.atcc.org) and Cellosaurus (web.expasy. org/cellosaurus/) both note that ECC-1 cells are actually Ishikawa contaminants. This assertion has been repeated by another study (8), but has never fully documented by anything beyond COmbined DNA Index System (CODIS) genotypes.

HEC50co. The 'co' sub-line was clonally derived from HEC50 cells in the laboratory of Dr Kimberly Leslie in the late 1990s (24). The parent cell line was established in 1975 from ascites obtained from a Japanese patient with a grade 3 endometrial adenocarcinoma (25). Transplants of HEC50 cells into nude mice result in poorly differentiated adenocarcinomas with papillary serous features (25). The HEC50co sub-line produces the same phenotype in mice (26). A thorough characterization of the sub-line has shown that HEC50co cells are consistent with type II endometrial cancer, which is aggressive and has a poor prognosis (12). This assignment was supported by another study that also confirmed p53 status as a null phenotype (27).

$K L E$. The KLE cell line was derived from a 68-year-old Caucasian patient at the Vincent Memorial Hospital in Boston, Massachusetts. The patient had previously been surgically treated for a grade II/IV endometrial adenocarcinoma but upon readmission was found to have a large, poorly differentiated adenocarcinoma extending into the parametrium (28). A portion of this tumor was the parent tissue of the cell line. KLE cells do not maintain either estrogen or progesterone receptors. The cell line was deposited in the ATCC soon after it was fully characterized and is still available (CRL-1622).

RL95-2. A 65-year-old, obese Caucasian patient presented at the University of Arizona Medical Center in 1980 with a grade 2 moderately differentiated adenosquamous carcinoma of the endometrium. Tumor epithelial cells were successfully isolated from the stromal and fibroblastic cells and cultured as the RL95-2 cell line (29). This cell line, which is positive for the estrogen receptor in both the cytosol and nucleus, is consistent with glandular epithelium on microscopic and biochemical criteria (29). The RL95-2 cell line is available from the ATCC (CRL-1671).

\section{Materials and methods}

Database of endometrial cancer cell lines. A list of endometrial cancer cell lines was compiled by mining the International Agency for Research on Cancer (IARC) TP53 database (www.iarc.fr) using the search term 'corpus uteri.'

Cell culture. The ECC-1, KLE and RL95-2 cells were purchased from the American Type Culture Collection, while Ishikawa cells and the HEC50 cells from which Hec50co originated were gifts from Dr Erlio Gurpide (New York University) (12). Ishikawa and Hec50co cells were cultured in DMEM supplemented with $10 \%$ fetal bovine serum (FBS) and $1 \%$ antibiotic [penicillin (p)/streptomycin (s)] (all from Gibco; Thermo Fisher Scientific, Inc.). KLE cells were cultured in RPMI 1640 (Gibco; Thermo Fisher Scientific, Inc.) supplemented with $10 \% \mathrm{FBS}$ and $1 \%$ p/s. ECC-1 cells were cultured RPMI 1640 supplemented with 5\% FBS and 1\% p/s. RL95-2 
cells were cultured in DMEM/F12 (Gibco; Thermo Fisher Scientific, Inc.) supplemented with $10 \%$ FBS and $1 \%$ p/s.

Nucleic acid purification and quality control $(Q C)$. Whole cell RNAs were purified using the mirVana RNA isolation kit according to manufacturer's recommendations (Thermo Fisher Scientific, Inc.). Yield and purity were assessed in the Genomics Division of the University of Iowa Institute for Human Genetics (IIHG) using an Agilent Model 2100 DNA Analyzer and a Trinean DropSense 16 spectrophotometer. Genomic DNA (gDNA) was purified using the DNeasy kit according to manufacturer's recommendations (Qiagen, Inc.). Yield and purity were determined using a NanoDrop 2000 spectrophotometer and horizontal gel electrophoresis $[1 \%$ agarose in $1 \mathrm{X}$ Tris/Borate/EDTA (TBE) buffer (0.13 M Tris, pH 7.6; $45 \mathrm{mM}$ boric acid; $2.5 \mathrm{mM}$ EDTA /Borate/EDTA)]. Gels were visualized on a uV transilluminator at $302 \mathrm{~nm}$ following staining in a $2 \%(\mathrm{v} / \mathrm{v})$ solution of ethidium bromide (Sigma).

Short tandem repeat (STR) genotyping. Quality control tested gDNA samples (100 ng per cell line) were submitted to Bio-Synthesis, Inc. (Cat. No. CL1003, www.biosyn.com), for 15 loci STR plus amelogenin genotyping. The STR panel is the core technology in the CODIS system genotyping system (30).

RNA sequencing. Cellular RNAs of sufficient quality, as determined by an RNA Integrity Number (31) $>9.0$ from the Agilent DNA Analyzer, were selected for RNA sequencing. Sequencing was carried out by the Genomics Division of the University of Iowa Institute for Human Genetics (IIHG). Transcription profiling was performed starting with $500 \mathrm{ng}$ total cellular RNA, which was fragmented, converted to cDNA and ligated to sequencing adaptors containing indexes; all steps were performed using the Illumina TruSeq stranded total RNA library preparation kit (Illumina, Inc.) per the manufacturer's protocol. Molar concentrations of the indexed libraries were measured using the Model 2100 Agilent Bioanalyzer and combined pooled in equimolar concentrations for sequencing. The concentration of the pools was measured using the Illumina Library Quantification kit (Kapa Biosystems; Roche Diagnostics) per the manufacturer's protocol and sequenced using the Illumina HiSeq 4000 genome sequencer and $150 \mathrm{bp}$ paired-end SBS chemistry per the manufacturer's protocol. RNA-seq reads were mapped and aligned to the human reference genome (version hg38) using STAR, a paired-end enabled algorithm (32). BAM files were produced after alignment.

MLH1 methylation status. Methylation of the MLH1 locus was shown in TCGA (https://portal.gdc.cancer.gov/) characterization of endometrial cancer to be nearly diagnostic of the MSI hypermutated type of tumor (13). With the same high quality gDNA that was submitted for STR genotyping, bisulfite conversions on all five cell lines were performed using the EZ DNA Methylation kit according to manufacturer's recommendation (Zymo Research Corp.). Converted bisDNA was then PCR amplified in the presence of 2X ZYMOTaq pre-mix (Zymo Research Corp.) with the following bisulfite-specific PCR primers in the promoter of the human (h)MLH1 gene per the manufacturer's protocol: hMLH1, Forward: 5'-GGA
GTGAAGGAGGTTACGGGTAAGT-3' and reverse: 5'-AAA AACGATAAA ACCCTATACCTAATCTATC-3'. These sequences, along with bisPCR conditions and both methylated and bis-converted control DNAs, are available from ZYMO Research (www.zymoresearch.com). Cycling conditions were $95^{\circ} \mathrm{C}$ for $10 \mathrm{~min}$ followed by 40 cycles of $95^{\circ} \mathrm{C}$ for $30 \mathrm{sec}, 59^{\circ} \mathrm{C}$ for $30 \mathrm{sec}$ and $72^{\circ} \mathrm{C}$ for one minute and a final extension step at $72^{\circ} \mathrm{C}$ for seven minutes. PCR amplicons were visualized on a $1.3 \%$ horizontal agarose gel following electrophoresis. The gel was visualized on a $\mathrm{uV}$ transilluminator at $302 \mathrm{~nm}$ following staining in a $2 \%(\mathrm{v} / \mathrm{v})$ solution of ethidium bromide (Sigma Chemical). Controls consist of bisulfite-converted human genomic DNA where $\mathrm{C} 1$ is completely methylated and $\mathrm{C} 2$ is a methylation control.

MSI. High molecular weight gDNA was purified from each cell line and QC assessed as aforementioned. Equal mass aliquots of gDNA (500 ng) were delivered to the Clinical Microbiology Laboratory of the University of Iowa Hospitals and Clinics whereupon MSI testing was carried out by multiplex PCR followed by fluorescence capillary electrophoresis, as is routinely performed for clinical testing by the Clinical Microbiology Laboratory at the University of Iowa Hospitals and Clinics. Following the 1997 National Cancer Institute consensus, each cell line was genotyped for the mononucleotide and dinucleotide repeat markers BAT25, BAT26, D2S123, D5S346 and D17S250 (33).

Variant calling. The reference genome used was hg38 with a $20-\mathrm{kb}$ bin size, as previously recommended (32). BAM files for each sample were used for mutation discovery and base calling against the human genome reference hg38 utilizing SAMtools and BCFtools for sorting and indexing (34). Results were annotated using ANNOVAR and formatted to display the number of mutations per gene and sample (32). Only non-synonymous somatic mutations were included. Data were cross-referenced to hg38 chromosomal coordinate in addition to base change.

$C N V$. Copy number was determined across the genome for each cell line from the RNA sequencing output. CNV was determined using SAMtools (version 1.7) and CopywriteR (version 2.20.0) using BAM files as the input $(34,35)$. SAMTools was used to sort and index BAM files to be used by CopywriteR. The reference genome used was hg38 with a 20 -kb bin size, as previously recommended (32). CopywriteR extracts copy number information from targeted sequencing by utilizing off-target reads, and can be used without reference and applied to sequencing data obtained from various techniques (35). To determine the copy number characteristics for each cell line, variation was measured as the standard deviation of $\log 2$ copy number both for specific genes and for chromosomal segments. The significance cut-off was $3 \mathrm{x}$ mean standard deviation for the entire genome and the cell lines were comparatively assessed based on this.

Homologous recombination repair (HRR). To assess the HRR status, RNA-sequencing data were analyzed for mutations in the following 12 genes (36): Breast cancer susceptibility protein (BRCA) 1, BRCA2, ataxia telangiectasia mutated (ATM), BRCA1 interacting protein C-terminal helicase 1 (BRIP1), 
Table I. Combined DNA Index system short-tandem repeat genotyping of the endometrial cancer cell lines in the present study. Data are presented consistent with the STR convention, whereby values reflect the number of repeats between primers at each locus on each chromosome, including the $\mathrm{X}$ chromosome (' $\mathrm{X}$ ').

\begin{tabular}{|c|c|c|c|c|c|c|c|c|c|c|}
\hline \multirow[b]{3}{*}{ Marker } & \multicolumn{10}{|c|}{ Cell line } \\
\hline & \multicolumn{2}{|c|}{ Ishikawa } & \multicolumn{2}{|c|}{ ECC-1 } & \multicolumn{2}{|c|}{ Hec50co } & \multicolumn{2}{|c|}{ KLE } & \multicolumn{2}{|c|}{ RL95-2 } \\
\hline & Korch & In-house & Korch & In-house & Korch & In-house & Korch & In-house & Korch & In-house \\
\hline CSF1PO & 11,12 & 11,12 & 11,12 & 11,12 & 9,12 & 9,12 & 13,14 & 13,14 & 10,11 & 10,11 \\
\hline D3S1358 & 17,18 & 16,16 & 16,17 & 16,17 & 15,16 & $15,16,17$ & 17,17 & 17,17 & 14,16 & 14,16 \\
\hline D5S818 & 10,11 & 9,10 & 10,11 & 10,11 & 8,8 & 8,8 & 9,12 & 9,12 & 10,11 & 10,11 \\
\hline D7S820 & 9,10 & 9,10 & 9,10 & 9,10 & 12,12 & 12,12 & 11,12 & 11,12 & 10,10 & 10,10 \\
\hline D8S1179 & 12,16 & 12,16 & 13,16 & 13,16 & 10,15 & 10,15 & 8,14 & 8,14 & 10,14 & 10,14 \\
\hline D13S317 & 9,12 & 9,13 & 9,12 & 9,12 & 9,9 & 9,9 & 12,12 & 12,12 & 8,12 & 8,12 \\
\hline D16S539 & 9,9 & 9,9 & 9,9 & 9,9 & 12,12 & 12,12 & 11,12 & 11,12 & 11,13 & 11,13 \\
\hline D18S51 & $12,13,22$ & $14,20,21$ & 12,19 & 12,19 & 14,14 & 14,14 & 13,17 & 13,17 & 10,14 & 10,14 \\
\hline D21S11 & 28,28 & 28,28 & 28,28 & 28,28 & 30,30 & 30,30 & 28,30 & 28,30 & 28,29 & 28,29 \\
\hline FGA & 21,21 & 21,21 & 21,21 & 21,21 & 20,20 & 20,20 & 23,25 & 23,25 & 20,22 & 20,22 \\
\hline THO1 & 9,10 & 9,10 & 9,10 & 9,10 & 9,9 & 9,9 & 6,7 & 6,7 & $9,9.3$ & $9,9.3$ \\
\hline TPOX & 8,8 & 8,8 & 8,8 & 8,8 & 9,9 & 9,9 & 8,11 & 8,11 & 8,8 & 8,8 \\
\hline vWA & 14,17 & 14,18 & 14,17 & 14,17 & 14,14 & 14,14 & 16,16 & 16,16 & 16,20 & 16,20 \\
\hline Amelogenin & $X$ & $\mathrm{X}$ & $\mathrm{X}$ & $\mathrm{X}$ & $\mathrm{X}$ & $\mathrm{X}$ & $\mathrm{X}$ & $\mathrm{X}$ & $\mathrm{X}$ & $\mathrm{X}$ \\
\hline
\end{tabular}

Korch, data reported by Korch et al (8); In-house, genotypes of the cells used in the present study.

checkpoint kinase 2 (CHEK2), Fanconia anemia complementation (FANC) group A (FANCA), FANC group I (FANCI), FANCM group M (FANCM), nibrin (NBN), RAD51 Paralog C (RAD51C), RAD51 Paralog D (RAD51D) and RAD51 Paralog L (RAD51L). The ClinVar database (www.ncbi.nlm. nih.gov/clinvar/), dbSNP (www.ncbi.nlm.nih.gov/snp/) and ARUP Laboratories (www.aruplab.com/) were assessed for prior reports of each detected mutation in these 12 loci.

\section{Results}

Comprehensive list of endometrial cancer cell lines. In 1999, Satyaswaroop compiled a characterization of 24 endometrial cancer cell lines which, at the time, represented virtually all of the cell lines in use in the world (37). Using the IARC TP53 database (www.iarc.fr) as a starting point, we have now assembled information on 127 putative endometrial cancer cell lines. Table SI presents basic information on these cell lines to the extent possible based on the original literature. Notably, these cell lines represent numerous ethnic groups as well as a wide range of histological types. The availability of each cell line is also noted where possible.

STR genotyping. The STR typing data are presented in Table I, comparing STR typing of the present (in-house) cells with those reported by Korch et al (8), the only published STR typing of endometrial cancer cell lines. Based on the STR data, it was confirmed that the cell models in our laboratory are correctly identified compared with the results of Korch et al (8). Ishikawa cells were consistent with the 3-H-12 sub-cell line documented by Nishida (21) as the sub-clone distributed after
1996. Based on the STR genotypes of Ishikawa and ECC-1 cells, these are likely the same cell line, as has been previously argued (8). It also appears that the ancestor of the current ECC-1 cell line is the 3-H-12 sub-cell line distributed after 1996. However, genomic profiling data shown below suggest that while Ishikawa and ECC-1 cells are very similar, they are not identical.

RNA sequencing. RNA sequencing output BAM files were aligned against Build 38 (GRCh38) of the human genome (hg38). DNA base calling differences between each cell line and the reference genome was then tabulated for each of the cell lines. In all, Ishikawa displayed 2,711 base calling differences compared with hg38, ECC-1 displayed 2,882, KLE displayed 1,488 base, RL95-2 displayed 2,756 and Hec50co displayed 1,508 (data not shown). As each base calling difference between the cell lines and the reference genome was identified both by base change and chromosome coordinate, all base calling differences shared by two or more of the cell lines were identified. It was observed that 274 base calling differences were shared by all five cell lines. Given the historical origins of the cell lines, it was assumed that it is unlikely that these base calling differences are anything more than variations in the hg38 reference genome as opposed to systematic mutations in endometrial cancer. The likelihood that cell lines from five unique individuals from different ethnicities and times would share consistent mutations compared to a sixth unique individual representing the human reference genome is small. Thus, accepting the possibility that potential shared mutations may be missed, base calling differences that were unique or shared by only two cell lines were further investigated. 
Table II. Mutation profile of the five cell lines for twelve commonly mutated loci in endometrial cancer.

\begin{tabular}{|c|c|c|c|c|c|c|c|}
\hline \multirow[b]{2}{*}{ Gene } & \multirow[b]{2}{*}{ Chr } & \multirow{2}{*}{$\begin{array}{l}\text { Mutation } \\
\text { frequency, \% }\end{array}$} & \multicolumn{5}{|c|}{ Cell line } \\
\hline & & & Ishikawa & ECC-1 & Hec50co & KLE & RL95-2 \\
\hline POLE & 12 & 11.21 & $\mathrm{P} 102 \mathrm{~S}^{\mathrm{a}}$ & $\mathrm{P} 102 \mathrm{~S}^{\mathrm{a}}$ & $\mathrm{Nd}^{\mathrm{b}}$ & $\mathrm{Nd}$ & $\mathrm{Nd}$ \\
\hline MLH1 & 3 & 2.59 & $\mathrm{Nd}$ & $\begin{array}{l}\mathrm{Nd} \\
\text { Promoter-Me }\end{array}$ & $\mathrm{Nd}$ & $\mathrm{Nd}$ & $\begin{array}{l}\mathrm{Nd} \\
\text { Promoter-Me }\end{array}$ \\
\hline TP53 & 17 & 28.88 & $\begin{array}{l}\text { M246V } \\
(\mathrm{rs} 483352695)^{\mathrm{d}}\end{array}$ & $\begin{array}{l}\text { M246V } \\
(\mathrm{rs} 483352695)^{\mathrm{d}}\end{array}$ & del G187-E224 & $\begin{array}{l}\mathrm{R} 175 \mathrm{H} \\
(\mathrm{rs} 28934578)^{\mathrm{d}}\end{array}$ & del V218 \\
\hline PTEN & 10 & 63.73 & E91fs-ter & E91fs-ter & $\mathrm{Nd}$ & $\mathrm{Nd}$ & T321fs-ter \\
\hline PIK3CA & 3 & 53.02 & $\mathrm{Nd}$ & $\mathrm{Nd}$ & $\mathrm{Nd}$ & $\mathrm{Nd}$ & $\mathrm{Nd}$ \\
\hline PIK3R 1 & 5 & 32.76 & L570P & L570P & $\begin{array}{l}\text { E469G } \\
\text { Y470D }\end{array}$ & $\mathrm{Nd}$ & $\begin{array}{l}\text { R386 } \\
\text { R639ter }\end{array}$ \\
\hline ARID1A & 1 & 33.62 & $\mathrm{Nd}$ & $\mathrm{Nd}$ & Y148ter & $\mathrm{Nd}$ & $\begin{array}{l}\text { L649fs } \\
\text { R693ter }\end{array}$ \\
\hline KRAS & 12 & 20.69 & $\mathrm{Nd}$ & $\mathrm{Nd}$ & $\begin{array}{l}\text { G12D } \\
(\mathrm{rs} 121913529)^{\mathrm{d}}\end{array}$ & $\mathrm{Nd}$ & $\mathrm{Nd}$ \\
\hline CTNNB1 & 3 & 29.74 & $\mathrm{Nd}$ & $\mathrm{Nd}$ & $\mathrm{Nd}$ & $\mathrm{Nd}$ & $\mathrm{Nd}$ \\
\hline FBXW7 & 4 & 16.81 & $\mathrm{Nd}$ & $\mathrm{Nd}$ & $\mathrm{Nd}$ & $\begin{array}{l}\text { R479Q } \\
(\text { rs866987936) }\end{array}$ & $\mathrm{Nd}$ \\
\hline PPP2R1A & 19 & 10.78 & $\mathrm{Nd}$ & $\mathrm{Nd}$ & $\begin{array}{l}\text { R183W } \\
(\text { rs1057519946) }\end{array}$ & $\mathrm{Nd}$ & $\mathrm{Nd}$ \\
\hline
\end{tabular}

Mutational frequency was determined based on The Cancer Genome data reported in Supplementary Data File S3.2 in reference (13). ${ }^{\mathrm{a} M u t a-}$ tion not in the exonuclease domain. ${ }^{b}$ No mutation detected from RNA sequencing. ${ }^{c}$ Methylation confirmed by bisulfite PCR. ${ }^{\mathrm{d}}$ Variant registered in dbSNP. Chr, chromosome; nd, no detected mutation; POLE, DNA polymerase $\varepsilon$ catalytic subunit A; MLH1, MutL homolog 1; PIK3CA, phosphatidylinositol 4,5-bisphosphate 3-kinase catalytic subunit $\alpha$ isoform; PIK3R1, phosphatidylinositol 3-kinase regulatory subunit $\alpha$; ARID1A, AT-rich interactive domain-containing protein 1A; CTNNB1, catenin $\beta-1$; FBXW7, F-box/WD repeat-containing protein 7; PPP2R1A, serine/threonine-protein phosphatase 2A $65 \mathrm{kDa}$ regulatory subunit $\mathrm{A} \alpha$ isoform.

TCGA-based endometrial cancer report listed a dozen genes as frequently mutated (13). The status of the cell lines for these loci is shown in Table II. Both Ishikawa and ECC-1 cells had a DNA polymerase $\varepsilon$ catalytic subunit A (POLE) mutation, but $\mathrm{P} 102 \mathrm{~S}$ is not in the proof-reading exonuclease domain at residues 268 to 471 (38) and therefore not an ultra-mutating event. All five cell lines harbored a TP53 mutation, and both Ishikawa and ECC-1 cells had an identical TP53 mutation, M246V. The precise phenotypic consequences of this variant are unknown, though the location of the mutant near the end of the L3 Loop of p53 that forms part of the crucial $\mathrm{Zn}^{2+}$ binding site (39) suggests that it is not neutral. Similarly, RL95-2 cells had an in-frame deletion of a single amino acid in TP53 (V218), the consequences of which are also unknown. On the other hand, Hec50co cells showed a large deletion in which the entirety of exon 6, G187 through E224, is absent, rendering these cells phenotypically p53 null. Lastly, KLE cells possessed one of the classic gain-of-function TP53 mutants, $\mathrm{R} 175 \mathrm{H}$, which subverts the tumor suppressor function of p53 into an oncomorphic one (40-42).

Three of the five cell lines are likely PTEN null. RL95-2 cells have a late frame-shift mutant, T321fs-ter, resulting in a premature termination in PTEN. A different PTEN frame-shift termination mutant, E91fs-ter, was observed in both Ishikawa and ECC-1 cells. Hec50co and RL95-2 cells had different mutations in phosphatidylinositol 3-kinase regulatory subunit $\alpha$ (PIK3RI) (E469G and Y470D in Hec50co; R386fs/R639ter in RL95-2) and AT-rich interactive domain-containing protein 1A (ARID1A) (Y148ter in Hec50co; L649fs/R693ter in RL95-2). Hec50co cells also possessed mutations in KRAS at G12D, serine/threonine-protein phosphatase 2A $65 \mathrm{kDa}$ regulatory subunit $\mathrm{A} \alpha$ isoform $(P P P 21 A)$ at $\mathrm{R} 183 \mathrm{~W}$ and F-box/WD repeat-containing protein 7 (FBXW7) at R479Q.

Ishikawa and ECC-1 cells also had an identical PIK3RI mutant, L570P, in addition to their identical POLE, TP53 and PTEN mutants. Comparing the entire base calling profile of the two cell lines (censored for base calling differences shared by three or more cell lines), Ishikawa and ECC-1 cells share 1,017 identical base calling differences with the hg38 reference (Fig. 1). This accounted for approximately one-half of all censored base calling differences. Meanwhile, RL95-2 cells, which have a similar number of base calling differences as Ishikawa and ECC-1 cells as aforementioned, shared only 12 identical base calling differences with Ishikawa cells (Fig. 1). These data substantiate the STR genotyping data that Ishikawa and ECC-1 cells are likely the same cell line.

MLH1 methylation status. Methylation-specific PCR of bisulfite-converted gDNA from each of the five cell lines plus two controls are shown in Fig. 2. Both the fully bisulfite-converted control (C1) and the methylation control (C2) demonstrated the expected 182-bp amplicon, indicating that the human MLH1 


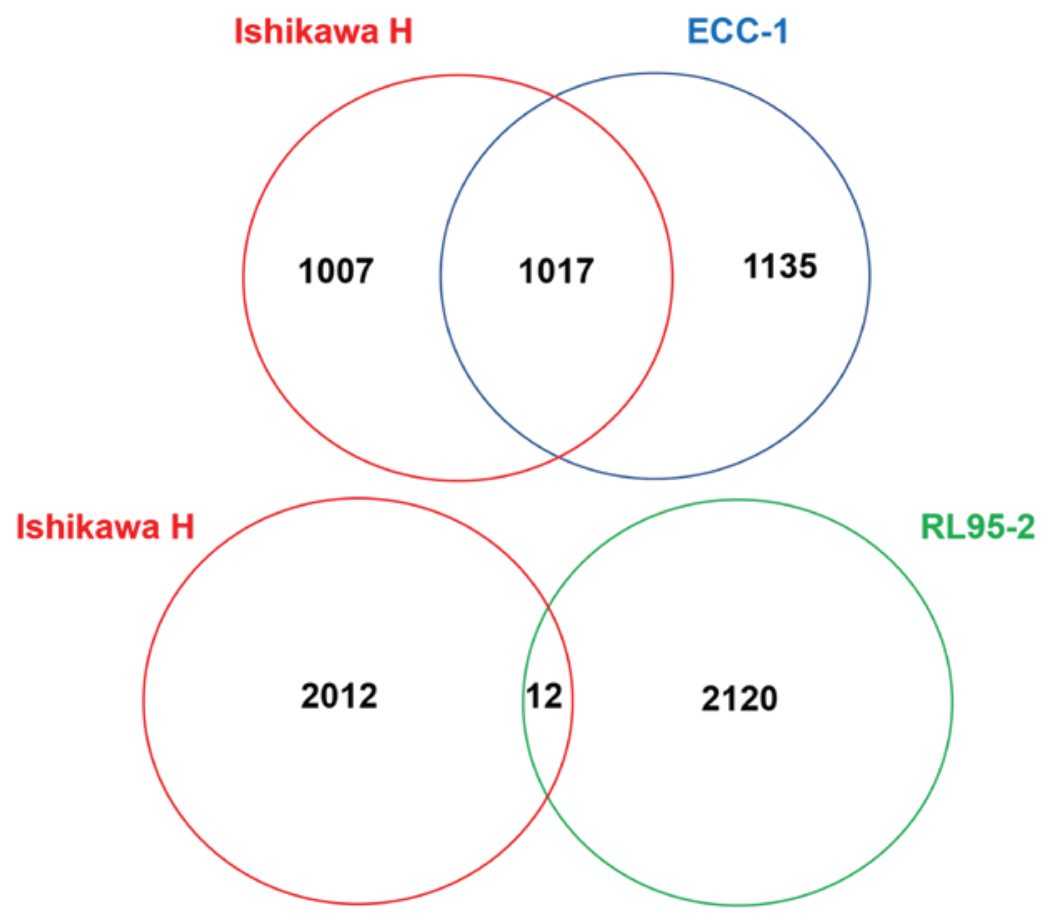

Figure 1. Shared censored base-calling differences between Ishikawa, ECC-1 and RL95-2 cells. The reference genome is the GRCh38 assembly.

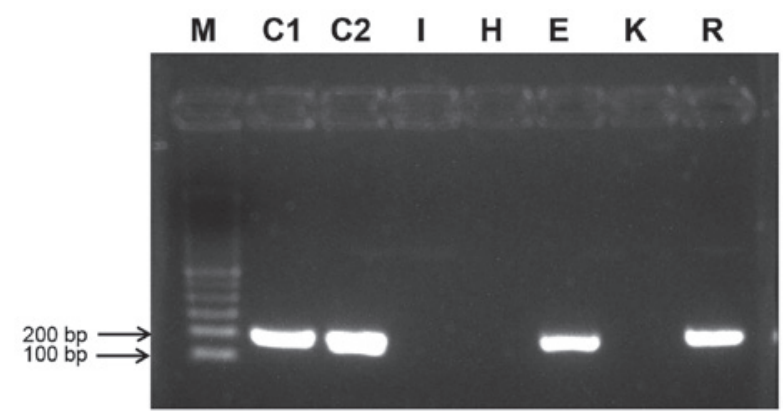

Figure 2. Methylation-specific PCR of the human MutL homolog promoter. The 182 amplicon is produced only when the promoter is methylated and, thus, inactive. $\mathrm{C} 1$ is a control composed of bisDNA, C2 is a bisulfate conversion experimental control. bisDNA, fully bisulfate-converted DNA I, Ishikawa bisDNA; H, Hec50co bisDNA; E, ECC-1 bisDNA; K, KLE bisDNA; R, RL95-2 bisDNA; M, marker.

promoter was methylated in these DNAs. Of the five cell lines, ECC-1 and RL95-2 produced an amplicon, indicating that their MLH1 promoter was methylated and thus inactive.

MSI. Results of MSI analysis are presented in Table III. Both Ishikawa and ECC-1 cells were classified as MSI-High, RL95-2 cells as MSI-Low and both Hec50co and KLE cells as MSI stable. The assignment of MSI-High status to Ishikawa and ECC-1 cells agrees with the conclusion offered by Korch et al (8) that the cell lines are of the same origin. It should be noted that Ishikawa but not ECC1 cells harbor a previously unreported MSH6 mutation, L398R, though the significance of this mutation to MSI status is not known.

$H R R$. The ability of cells to carry out HRR has been linked to breast cancer susceptibility protein (BRCA) mutation status (43). Other loci known to influence this ability include ataxia telangiectasia mutated (ATM), genes associated with Fanconi anemia (for example FANCD2) and the RAD51 family of recombinases (36). RNA sequencing data were searched for mutations in 12 relevant loci. In total, 18 mutations were identified across the five cell lines (Table IV). Using assessments of the likely effect of each mutation available in the ClinVar database, dbSNP and ARUP Laboratories, it is likely that all five cell lines are HRR proficient, although the splice site BRCA1 mutant observed in ECC-1 cells suggested that ECC-1 may be HRR deficient; however, additional studies are necessary to directly assess HR proficiency.

$C N V$. The overall pattern of CNV for both chromosomal segments and individual genes is presented in Fig. 3A and B. In total, 75,640 chromosome segments were analyzed and 404 of these reached the cut-off of 3x mean standard deviation (Fig. 3A). Similarly, 28,918 genes were assessed, with 117 of these at or above the 3x mean standard deviation threshold. The identity of these 117 genes along with their chromosome location and relative copy numbers are shown in Fig. 3C and Table SII. In terms of simply comparing the cell lines to each other, it was clear that Ishikawa cells are more prone to copy number loss compared with the other cell lines, whereas RL95-2 cells would be termed 'copy number high' according to TCGA classification schemes (13). There was a similarity in copy number patterns between Ishikawa and ECC-1 cells (Fig. 3C), but this is not as notable as it is with other aforementioned analysis (STR genotyping).

Overall, CNVs are not as pronounced in endometrial cancer as with other cancer types. Notable exceptions include focal amplifications of the MYC proto-oncogene (8q24.12), Erb-B2 receptor tyrosine kinase (17q12) and cyclin E1 (19q12), which appear to be characteristic of serous endometrial cancer (13-15). However, none of these were observed in the five present cell 
Table III. MSI in the five endometrial cancer cell lines.

\begin{tabular}{lrrrrrr}
\hline & \multicolumn{5}{c}{ Marker } & \\
\cline { 2 - 5 } Cell line & BAT26 & \multicolumn{1}{c}{ BAT25 } & D2S123 & D5S346 & D17S250 & Assignment \\
\hline Ishikawa & 103,104 & $114,115,120$ & 94,115 & 82,90 & 128,136 & MSI-High \\
ECC-1 & 105 & $114,115,118$ & 97,119 & 82,88 & 126,134 & MSI-High \\
Hec50c0 & 117 & 123 & 101,109 & 82 & 132 & MSI-Stable \\
KLE & 117,118 & 122,123 & 104,106 & 86,94 & 132 & MSI-Stable \\
RL95-2 & 107 & 117,120 & 99,177 & 86 & 151,155 & MSI-Low \\
\hline
\end{tabular}

MSI, microsatellite instability.

Table IV. Mutation profile of HRR genes in the five endometrial cancer cell lines.

\begin{tabular}{|c|c|c|c|c|c|}
\hline \multirow[b]{2}{*}{ Marker } & \multicolumn{5}{|c|}{ Cell line } \\
\hline & Ishikawa & ECC-1 & Hec50co & KLE & RL95-2 \\
\hline BRCA1 & $\begin{array}{l}\text { P871L } \\
\text { S1634G }\end{array}$ & $\begin{array}{l}\text { P871L } \\
\text { S1634G } \\
\text { 17:4307705 splice }\end{array}$ & $\mathrm{Nd}$ & $\mathrm{Nd}$ & $\mathrm{Nd}$ \\
\hline BRCA2 & $\mathrm{N} 289 \mathrm{H}$ & $\mathrm{N} 289 \mathrm{H}$ & $\mathrm{Nd}$ & A2852fs & K2551E \\
\hline ATM & $\mathrm{Nd}$ & R1312fs & $\mathrm{Nd}$ & $\mathrm{Nd}$ & $\mathrm{Nd}$ \\
\hline BRIP1 & $\mathrm{Nd}$ & $\mathrm{Nd}$ & $\mathrm{Nd}$ & $\mathrm{Nd}$ & L195P \\
\hline CHEK2 & $\mathrm{Nd}$ & $\mathrm{Nd}$ & $\mathrm{Nd}$ & $\mathrm{Nd}$ & $\mathrm{Nd}$ \\
\hline FANCA & $\mathrm{Nd}$ & $\mathrm{Nd}$ & $\mathrm{Nd}$ & $\mathrm{Nd}$ & $\mathrm{Nd}$ \\
\hline FANCI & L781R & $\mathrm{Nd}$ & S371G & $\mathrm{Nd}$ & $\mathrm{Nd}$ \\
\hline FANCM & R798G & $\mathrm{H} 742 \mathrm{Y}$ & $\begin{array}{l}\text { I1460V } \\
\text { P1812A }\end{array}$ & $\mathrm{Nd}$ & $\mathrm{Nd}$ \\
\hline NBN & $\mathrm{Nd}$ & $\mathrm{Nd}$ & $\mathrm{Nd}$ & $\mathrm{Nd}$ & $\mathrm{Nd}$ \\
\hline RAD51C & $\mathrm{Nd}$ & $\mathrm{Nd}$ & $\mathrm{Nd}$ & $\mathrm{Nd}$ & $\mathrm{Nd}$ \\
\hline RAD51D & $\mathrm{Nd}$ & $\mathrm{Nd}$ & $\mathrm{Nd}$ & $\mathrm{Nd}$ & $\mathrm{Nd}$ \\
\hline RAD51L & $\mathrm{Nd}$ & $\mathrm{Nd}$ & R154Q & $\mathrm{Nd}$ & $\mathrm{Nd}$ \\
\hline
\end{tabular}

nd, no detected mutation; BRCA, breast cancer susceptibility protein; ATM, ataxia telangiectasia mutated; BRIP1, BRCA1 interacting protein C-terminal helicase 1; CHEK2, checkpoint kinase 2; FANC, Fanconia anemia complementation; FANCA, FANC group A; FANCI, FANC group I; FANCA, FANCM group M; NBN, nibrin; RAD51C, RAD51 Paralog C; RAD51D, RAD51 Paralog D; RAD51L, RAD51 Paralog L.

lines. On the other hand, a substantial region of chromosome 4, 4p16-4p15, was amplified in Hec50co and RL95-2 cells (Fig. 3C). This region contains several significantly altered loci surrounding the locus fibroblast growth factor receptor 3 (4p16.3), which has previously been noted in endometrial cancer (13).

Another locus, insulin-like growth factor 1 receptor (IGF1R; $15 q 26.3)$ has been identified as a focal amplification in endometrial cancer $(13,15)$. In the present study, the $15 q 26.3$ region was amplified in KLE and ECC-1 cells but did not reach significance as established by the cut-off of $3 \mathrm{x}$ the mean standard deviation (Fig. 3C). Meanwhile, arrestin domain containing 4 (ARRDC4; 15q26.2) did reach significance in ECC-1 cells (Fig. 3C). This was one of a number of CNVs where Ishikawa and ECC-1 cells did not present the same profile (Fig. 3C), suggesting that prolonged culture has led to development of unique features that are not present in the original Ishikawa cells.

\section{Discussion}

The present study attempted a genomic characterization of five commonly used endometrial cancer cell lines: Ishikawa, ECC-1, Hec50co, KLE And RL95-2 through a combination of STR genotyping, RNA sequencing, methylation-specific PCR and MSI testing. The data presented showed that these cells are a mixture of characteristics. None of these cell lines are wholly representative of any one of the four TGCA-based clusters (13-16). This does not come as a surprise since several datasets in TCGA and Pan-Cancer Analysis of Whole Genomes have suggested that any individual cell line or tumor will likely present characteristics of two or more clusters, thus making such static categorizations problematic (12-16). For example, the current study demonstrated that the Ishikawa cell line contains a non-activating POLE mutation a TP53 mutation of 

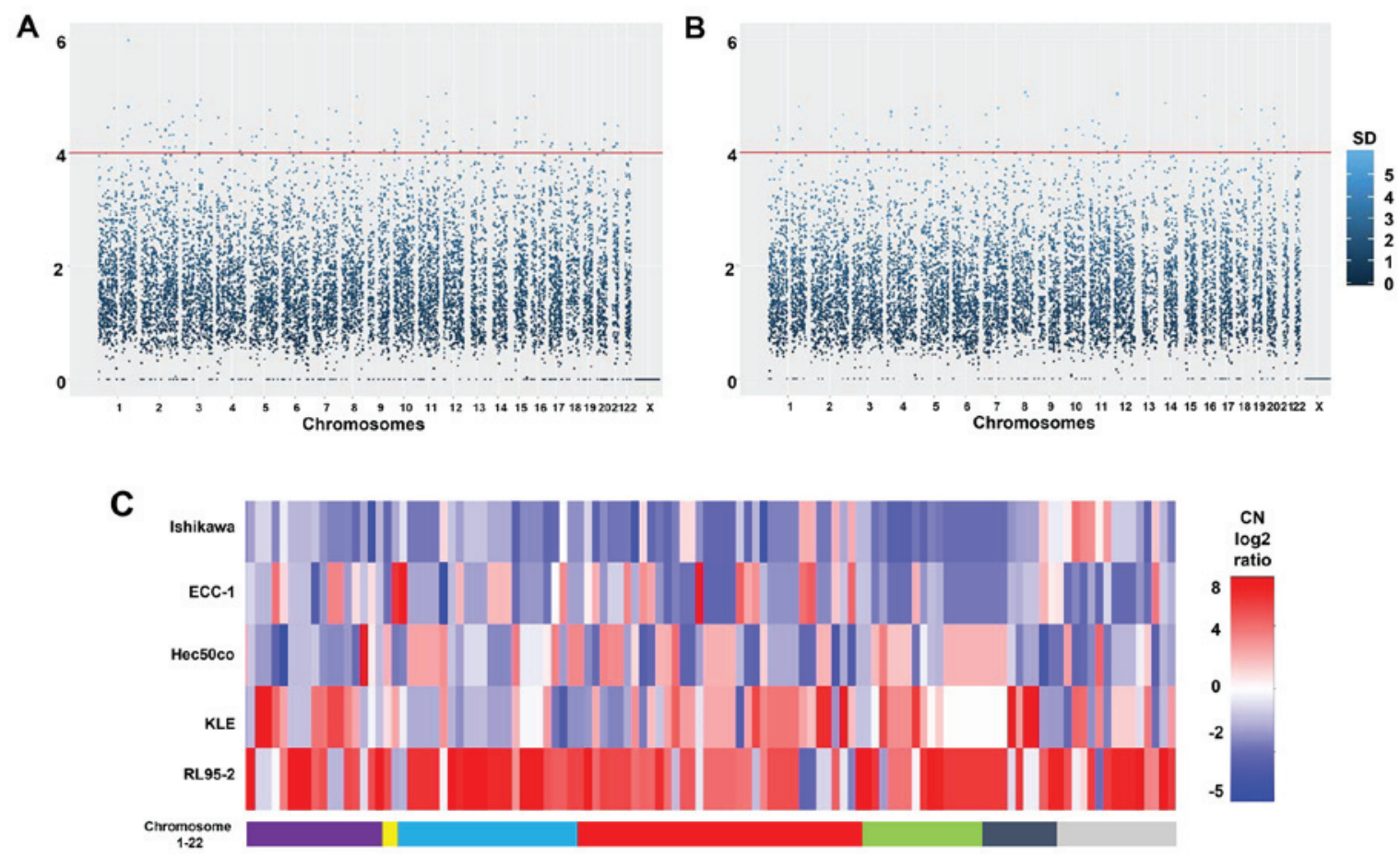

Figure 3. RNA sequencing-based copy number analysis of the five endometrial cancer cell lines. (A) Copy number expressed as standard deviation of log2 ratio of chromosome coordinates for all five cell lines combined. The cut-off (red line) was set at 3x mean SD over the entire genome ( $>99$ th percentile). Of 75,640 chromosome segments analyzed, 404 reached or exceeded the cut-off value. (B) Same analysis as in (A) but for 28, 918 individual genes for all five cell lines combined. Of these genes, 117 reached or exceeded the cut-off significance value. (C) A comparative heat map of $\log 2$ ratio for the 117 individual genes for each of the five cell lines expressed separately. The 117 gene list is presented in Table SII along with the relevant chromosomal regions.

unknown function and a wild-type PTEN gene and is MSI-High and copy number low. Also, Ishikawa cells originated from a patient with a well differentiated grade 2 adenocarcinoma (19). Thus, Ishikawa cells display characteristics of cluster 2, 3 and 4 but in vivo will form endometrioid tumors in mice (21). A similar set of inconclusive arguments can be made for each of the five cell lines. For example, RL95-2 cells do not have a POLE mutation but do have a PTEN termination mutant, are MSI-Low and are copy number high. These features would place them closest to cluster 4, but they originated from a grade 2 moderately differentiated adenosquamous tumor, not a serous adenocarcinoma (29). KLE cells are wild-type for POLE, MSI-Stable and possess a well-known TP53 gain-of-function mutation. The tumor from which they originated was a poorly differentiated grade 3 adenocarcinoma, though precise histology was not provided (28). However, KLE cells are also close to cluster 4 . Finally, Hec50co cells form serous adenocarcinomas in mice (26), are MSI-Stable and p53 null. These cells have long been regarded as the archetype of the former type II endometrial carcinoma (12). While their copy number profile is intermediate among these cell lines, their other features of MSI-Stable and TP53-mutated best place them in cluster 4 .

Finally, the similarities displayed in the present study between ECC-1 and Ishikawa cells support the notion that ECC-1 cells are, in fact, Ishikawa cells. In addition to data presented herein, Korch et al provided data from two additional loci, Penta $\mathrm{C}$ and Penta D, that indicates these cells came from the same individual (8). However, there were differences that warrant regarding ECC-1 cells as similar but not identical to Ishikawa cells. Apart from the mutation profile itself, both Ishikawa and ECC-1 cells are MSI-positive, yet Ishikawa cells have an unmethylated MLH1 promoter while ECC-1 cells have a methylated promotor. There is a well-known association between MSI and MLH1 hypermethylation $(44,45)$; however, such inconsistencies have been previously reported. Endometrial tumors that are MSI-positive and MLH1 unmethylated have been identified (46). In such tumors there are inactivating mutations in the MutS homolog 6 (MSH6) gene (42). Ishikawa cells do display a previously unreported MSH6 mutation, L398R. This is not seen in ECC-1 cells; however, whether or not this mutation can explain the MLH1 methylation discrepancy between the two cell lines is unknown. Rather than simply dismissing ECC-1 cells as an Ishikawa contaminant as commercial sources have done, it might be useful to examine the differences between the two cell lines in order to assess the effect of those differences against a background of genomic similarity.

Of course, the endometrial cancer cell line story does not end with these five cell lines. The information on an additional 122 endometrial cancer cell lines was assembled. While the five cell lines reported in the present study may meet the criteria for both mechanistic and pre-clinical endometrial cancer models, the existence of numerous other, less well characterized, cell lines suggests that better representatives may be available. Further, other cell lines may be better suited to different types of studies relevant to endometrial cancer. For example, recent publications have documented the fact that, while endometrial cancer incidence in general is on the rise, the cancer that is seen in African American women seems to be of a different nature in terms of incidence, histology and prognosis $(3,47)$. Accordingly, the 22 uterine serous papillary carcinoma ARK serous cells originated from a population of patients, among whom 13 were 
identified as Caucasian and nine were identified as African American $(48,49)$. However, it is not clear if these cells are immortalized or are commercially available. Perhaps these or other novel cell lines could be used help improve our understanding of how the cancer afflicting African American women is distinct from endometrial cancer in other ethnic groups, and if those differences could be used to clinical advantage as has recently been suggested (47). Additionally, the present study indicated that, while the majority of endometrial cancer cell lines originate from adenocarcinomas, other histological types are in short supply, with only six cell lines originating from patients diagnosed with a malignant mixed Müllerian tumor, three from a clear cell histology and nine sarcomas. Among the 122 endometrial cancer cell lines for which we provide information herein, the original histology and source information is often lacking or confusing. Therefore, it would benefit the study of endometrial cancer if more detailed information were to be made available along with expanded genomic information. Ultimately, this would provide investigators with a wider field from which to select cells for in vitro pre-clinical studies.

In conclusion, the present study provides a comprehensive characterization of the five most commonly studied endometrial cancer cell lines, leveraging technological advances in high-throughput sequencing to define mutations, copy number variations and HRR proficiency. The data indicated that these cell lines do not cleanly fit into any of the four clusters as defined by TCGA, highlighting the limitation of studying cell lines that have been immortalized and passaged for, in several cases, decades. Therefore, researchers should be aware of the limitations associated with studies of cancer cells cultured in monolayers (2-dimensional) as was performed in the present study. Future studies in endometrial cancer could be expanded to 3-dimensional models using fresh patient tumors, as our group has recently reported using organoid models for ovarian cancer (50).

\section{Acknowledgements}

Not applicable.

\section{Funding}

The study was funded by The National Institutes of Health/National Cancer Institute (grant nos. R01 CA99908 and R01 CA184101) and by The Basic Research Fund of the University of Iowa Carver College of Medicine Department of Obstetrics and Gynecology.

\section{Availability of data and materials}

RNA sequencing data have been deposited in the National Institutes of Health Gene Expression Omnibus (GEO [https://www.ncbi.nlm.nih.gov/geo/]), (accession number GSE151207). The additional datasets analyzed during the current study are available from The Cancer Genome Atlas [https://portal.gdc.cancer.gov/], International Agency for Research on Cancer TP53 repositories [www.iarc.fr], ClinVar database [www.ncbi.nlm.nih.gov/clinvar/], dbSNP [www.ncbi.nlm.nih.gov/snp/] and ARUP Laboratories [www.aruplab.com/].

\section{Authors' contributions}

EJD carried out the cell cultures, purified total cellular RNAs and genomic DNAs, performed the bisulfite conversions and bisPCRs, and was a major contributor in writing the manuscript. JGB analyzed and interpreted the RNA sequencing data and was a major contributor in writing the manuscript. KWT was a major contributor in study design, data analysis and writing and editing the manuscript. KKL was the senior investigator, was the recipient of the National Institutes of Health grants and was a major contributor in conceptualization of the study, data analysis and writing the manuscript. All authors read and approved the final manuscript.

\section{Ethics approval and consent to participate}

Not applicable.

\section{Patient consent for publication}

Not applicable.

\section{Competing interests}

KWT is a co-founder of Immortagen, Inc. All other authors certify that they have no affiliations with or involvement in any organization or entity with any financial interests in the subject matter or materials discussed in this manuscript.

\section{References}

1. Ferlay J, Soerjomataram I, Dikshit R, Eser S, Mathers C, Rebelo M, Parkin DM, Forman D and Bray F: Cancer incidence and mortality worldwide: Sources, methods and major patterns in GLOBOCAN 2012. Int J Cancer 136: E359-E386, 2015.

2. Siegel R, Naishadham D and Jemal A: Cancer statistics, 2013. CA Cancer J Clin 63: 11-30, 2013.

3. Cote ML, Ruterbusch JJ, Olson SH, Lu K and Ali-Fehmi R: The growing burden of endometrial cancer: A major racial disparity affecting black women. Cancer Epidemiol Biomarkers Prev 24: 1407-1415, 2015.

4. Sheikh MA, Althouse AD, Freese KE, Soisson S, Edwards RP, Welburn S, Sukumvanich P, Comerci J, Kelley J, LaPorte RE and Linkov F: USA endometrial cancer projections to 2030: Should we be concerned? Future Oncol 10: 2561-2568, 2014.

5. Gey GO: Tissue culture studies of the proliferative capacity of cervical carcinoma and normal epithelium. Cancer Res 12: 264-265, 1952.

6. Borrell B: How accurate are cancer cell lines? Nature 463: 858, 2010.

7. Gillet JP, Calcagno AM, Varma S, Marino M, Green LJ, Vora MI, Patel C, Orina JN, Eliseeva TA, Singal V, et al: Redefining the relevance of established cancer cell lines to the study of mechanisms of clinical Anti-cancer drug resistance. Proc Natl Acad Sci USA 108: 18708-18713, 2011.

8. Korch C, Spillman MA, Jackson TA, Jacobsen BM, Murphy SK, Lessey BA, Jordan VC and Bradford AP: DNA profiling analysis of endometrial and ovarian cell lines reveals misidentification, redundancy and contamination. Gynecol Oncol 127: 241-248, 2012.

9. Domcke S, Sinha R, Levine DA, Sander C and Schultz N: Evaluating cell lines as tumour models by comparison of genomic profiles. Nat Commun 4: 2126, 2013.

10. Anglesio MS, Wiegand KC, Melnyk N, Chow C, Salamanca C, Prentice LM, Senz J, Yang W, Spillman MA, Cochrane DR, et al: Type-specific cell line models for type-specific ovarian cancer research. PLoS One 8: e72162, 2013.

11. Beaufort CM, Helmijr JC, Piskorz AM, Hoogstraat M, Ruigrok-Ritstier K, Besselink N, Murtaza M, van IJcken WF, Heine AA, Smid M, et al: Ovarian cancer cell line panel (OCCP): Clinical importance of in vitro morphological subtypes. PLoS One 9: e103988, 2014. 
12. Albitar L, Pickett G, Morgan M, Davies S and Leslie KK: Models representing type I and type II human endometrial cancers: Ishikawa $\mathrm{H}$ and Hec50co cells. Gynecol Oncol 106: 52-64, 2007.

13. Cancer Genome Atlas Research Network; Kandoth C, Schultz N, Cherniack AD, Akbani R, Liu Y, Shen H, Robertson AG, Pashtan I, Shen R, et al: Integrated genomic characterization of endometrial carcinoma. Nature 497: 67-73, 2013

14. Murali R, Soslow RA and Weigelt B: Classification of endometrial carcinoma: More than two types. Lancet Oncol 15: e268-e278, 2014.

15. Berger AC, Korkut A, Kanchi RS, Hegde AM, Lenoir W, Liu W, Liu Y, Fan H, Shen H, Ravikumar V, et al: A Comprehensive Pan-cancer molecular study of gynecologic and breast cancers. Cancer Cell 33: 690-705.e9, 2018.

16. Hoadley KA, Yau C, Hinoue T, Wolf DM,Lazar AJ, Drill E, Shen R, Taylor AM, Cherniack AD, Thorsson V, et al: Cell-of-Origin patterns dominate the molecular classification of 10,000 tumors from 33 types of cancer. Cell 173: 291-304.e6, 2018.

17. Bairoch A: The cellosaurus, a cell-line knowledge resource. J Biomol Tech 29: 25-38, 2018.

18. Robin T, Capes-Davis A and Bairoch A: CLASTR: The cellosaurus STR similarity search tool-A precious help for cell line authentication. Int J Cancer 146: 1299-1306, 2020.

19. Nishida M, Kasahara K, Kaneko M, Iwasaki H and Hayashi K Establishment of a new human endometrial adenocarcinoma cell line, Ishikawa cells, containing estrogen and progesterone receptors. Nihon Sanka Fujinka Gakkai Zasshi 37: 1103-1111, 1985 (In Japanese).

20. Nishida M, Kasahara K, Oki A, Satoh T, Arai Y and Kubo T: Establishment of eighteen clones of Ishikawa cells. Hum Cell 9: 109-116, 1996.

21. Nishida M: The Ishikawa cells from birth to the present. Hum Cell 15: 104-117, 2002.

22. Satyaswaroop PG, Zaino RJ and Mortel R: Human endometrial adenocarcinoma transplanted into nude mice: Growth regulation by estradiol. Science 219: 58-60, 1983 .

23. Mo B, Vendrov AE, Palomino WA, DuPont BR, Apparao KB and Lessey BA: ECC-1 cells: A well-differentiated Steroid-responsive endometrial cell line with characteristics of luminal epithelium. Biol Reprod 75: 387-394, 2006.

24. Dai D, Wolf DM, Litman ES, White MJ and Leslie KK: Progesterone inhibits human endometrial cancer cell growth and invasiveness: Down-regulation of cellular adhesion molecules through progesterone B receptors. Cancer Res 62: 881-886, 2002.

25. Kuramoto H, Nishida M, Morisawa T, Hamano M, Hata H, Kato Y, Ohno E and Iida T: Establishment and characterization of human endometrial cancer cell lines. Ann N Y Acad Sci 622: 402-421, 1991.

26. Dai D, Albitar L, Nguyen T, Laidler LL, Singh M and Leslie KK: A therapeutic model for advanced endometrial cancer: Systemic progestin in combination with local adenoviral-mediated progesterone receptor expression. Mol Cancer Ther 4: 169-175, 2005.

27. Liu Z, Wan G, Heaphy C, Bisoffi M, Griffith JK and Hu CA: A novel loss-of-function mutation in TP53 in an endometrial cancer cell line and uterine papillary serous carcinoma model. Mol Cell Biochem 297: 179-187, 2007.

28. Richardson GS, Dickersin GR, Atkins L, MacLaughlin DT, Raam S, Merk LP and Bradley FM: KLE: A cell line with defective estrogen receptor derived from undifferentiated endometrial cancer. Gynecol Oncol 17: 213-230, 1984.

29. Way DL, Grosso DS, Davis JR, Surwit EA and Christian CD: Characterization of a new human endometrial carcinoma (RL95-2) established in tissue culture. In Vitro 19: 147-158, 1983.

30. Cabrera CM, Cobo F, Nieto A, Cortés JL, Montes RM, Catalina P and Concha A: Identity tests: Determination of cell line Cross-contamination. Cytotechnology 51: 45-50, 2006.

31. Schroeder A, Mueller O, Stocker S, Salowsky R, Leiber M, Gassmann M, Lightfoot S, Menzel W, Granzow M and Ragg T: The RIN: An RNA integrity number for assigning integrity values to RNA measurements. BMC Mol Biol 7: 3, 2006.

32. Dobin A, Davis CA, Schlesinger F, Drenkow J, Zaleski C, Jha S Batut P, Chaisson M and Gingeras TR: STAR: Ultrafast universal RNA-seq aligner. Bioinformatics 29: 15-21, 2013.

33. Boland CR, Thibodeau SN, Hamilton SR, Sidransky D, Eshleman JR, Burt RW, Meltzer SJ, Rodriguez-Bigas MA, Fodde R, Ranzani GN and Srivastava S: A National cancer institute Workshop on microsatellite instability for cancer detection and familial predisposition: Development of international criteria for the determination of microsatellite instability in colorectal cancer. Cancer Res 58: 5248-5257, 1998.
34. Li H, Handsaker B, Wysoker A, Fennell T, Ruan J, Homer N, Marth G, Abecasis G and Durbin R; 1000 Genome Project Data Processing Subgroup: The sequence Alignment/Map format and SAMtools. Bioinformatics 25: 2078-2079, 2009.

35. Kuilman T, Velds A, Kemper K, Ranzani M, Bombardelli L, Hoogstraat M, Nevedomskaya E, Xu G, de Ruiter J, Lolkema MP, et al: CopywriteR: DNA copy number detection from off-target sequence data. Genome Biol 16: 49, 2015

36. Hoppe MM, Sundar R, Tan DSP and Jeyasekharan AD: Biomarkers for homologous recombination deficiency in cancer. J Natl Cancer Inst 110: 704-713, 2018.

37. Satyaswaroop PG: Endometrial Cancer. In: Human cell culture. Masters JRW and Palsson B (eds). Springer, Dordrecht, pp71-78, 1999.

38. van Gool IC, Bosse T and Church DN: POLE proofreading mutation, immune response and prognosis in endometrial cancer. Oncoimmunology 5: e1072675, 2015.

39. Chen Y, Dey R and Chen L: Crystal structure of the p53 core domain bound to a full consensus site as a self-assembled tetramer. Structure 18: 246-256, 2010.

40. Brachova P, Thiel KW and Leslie KK: The consequence of oncomorphic TP53 mutations in ovarian cancer. Int J Mol Sci 14: 19257-19275, 2013.

41. Brachova P, Mueting SR, Devor EJ and Leslie KK: Oncomorphic TP53 mutations in gynecologic cancers lose the normal protein:protein interactions with the microRNA microprocessing complex. J Cancer Ther 5: 506-516, 2014.

42. Brachova P, Mueting SR, Carlson MJ, Goodheart MJ, Button AM, Mott SL, Dai D, Thiel KW, Devor EJ and Leslie KK: TP53 oncomorphic mutations predict resistance to platinum and Taxane-based standard chemotherapy in patients diagnosed with advanced serous ovarian carcinoma. Int J Oncol 46: 607-618, 2015.

43. Walsh CS: Two decades beyond BRCA1/2: Homologous recombination, hereditary cancer risk and a target for ovarian cancer therapy. Gynecol Oncol 137: 343-350, 2015.

44. Esteller M, Levine R, Baylin SB, Ellenson LH and Herman JG: MLH1 promoter hypermethylation is associated with the microsatellite instability phenotype in sporadic endometrial carcinomas. Oncogene 17: 2413-2417, 1998.

45. Simpkins SB, Bocker T, Swisher EM, Mutch DG, Gersell DJ, Kovatich AJ, Palazzo JP, Fishel R and Goodfellow PJ: MLH1 promoter methylation and gene silencing is the primary cause of microsatellite instability in sporadic endometrial cancers. Hum Mol Genet 8: 661-666, 1999.

46. Goodfellow PJ, Buttin BM, Herzog TJ, Rader JS, Gibb RK, Swisher E, Look K, Walls KC, Fan MY and Mutch DG: Prevalence of defective DNA mismatch repair and MSH6 mutation in an unselected series of endometrial cancers. Proc Nat Acad Sci USA 100: 5908-5913, 2003.

47. DubilEA, Tian C, Wang G, Tarney CM, Bateman NW, Levine DA, Conrads TP, Hamilton CA, Maxwell GL and Darcy KM: Racial disparities in molecular subtypes of endometrial cancer. Gynecol Oncol 149: 106-116, 2018

48. Varughese J, Cocco E, Bellone S, de Leon M, Bellone M, Todeschini P, Schwartz PE, Rutherford TJ, Pecorelli S and Santin AD: Uterine serous papillary carcinomas overexpress human trophoblast-cell-surface marker (Trop-2) and are highly sensitive to immunotherapy with hRS7, a humanized anti-Trop-2 monoclonal antibody. Cancer 117: 3163-3172, 2011

49. English DP, Bellone S, Cocco E, Bortolomai I, Pecorelli S, Lopez S, Silasi DA, Schwartz PE, Rutherford T and Santin AD: Oncogenic PIK3CA gene mutations and HER2/neu gene amplifications determine the sensitivity of uterine serous carcinoma cell lines to GDC-0980, a selective inhibitor of Class I PI3 kinase and mTOR kinase (TORC1/2). Am J Obstet Gynecol 209: 465. e1-e9, 2013

50. Bi J, Thiel KW, Litman JM, Zhang Y, Devor EJ, Newtson AM, Schnieders MJ, Gonzalez Bosquet J and Leslie KK: Characterization of a TP53 somatic variant of unknown function from an ovarian cancer patient using organoid culture and computational modeling. Clin Obstet Gynecol 63: 109-119, 2020. 